\title{
Flexibilizando definições geométricas
}

\author{
Julia Schaetzle Wrobel \\ Universidade Federal do Espírito Santo \\ juliasw@gmail.com
}

\section{Thais Helena Nakassima Morosini \\ Centro Educacional Radier \\ thaismorosini@gmail.com}

\begin{abstract}
Resumo
Trata-se do relato de experiência de uma oficina sobre definições de polígonos e poliedros. Partindo do conhecimento dos alunos, verificamos definições incompletas e inconsistentes. Em uma veia investigativa, discutimos a necessidade de definição rigorosa, embora não absoluta. Trabalhamos os polígonos e poliedros estrelados, em definições e origami. Essa abordagem facilita a aprendizagem, permitindo a identificação do sólido e suas características a partir da manipulação de materiais concretos, e explora as potencialidades do Origami para o ensino da Matemática.
\end{abstract}

Palavras-chave: Polígonos. Poliedros. Poliedros Estrelados. Origami. Investigação.

\section{Adjusting geometric definitions}

\begin{abstract}
This article shows the experience in a workshop to discuss definitions of polygons and polyhedra. The students present incomplete and inconsistent definitions. We discussed the need for rigorous but not absolute definitions and we worked with star polygons and polyhedral, their definitions and constructions using modular origami technique. This approach facilitates learning, enables the identification of the solid and its features from the manipulation of concrete materials, and explores the potential of origami as a tool for teaching mathematics.
\end{abstract}

Keywords: Polygons. Polyhedra. Star Polyhedra. Origami. Investigation.

\section{Introdução}

Durante o estudo sobre Poliedros Regulares em um curso de formação de professores, um aluno apresentou a seguinte questão: existe poliedro regular não convexo? A pergunta foi seguida de argumentações que concluíram que não existiam. Segundo um dos alunos, para o poliedro ser não 
convexo, deveríamos retirar uma parte do sólido convexo, deixando uma reentrância. Essa ação, de tirar uma parte, faria com que as faces não fossem mais congruentes. Nenhum aluno, naquele momento, pensou em tirar partes iguais de todas as faces.

O ponto de partida das autoras para responder a questão sobre existência de poliedro regular não convexo foi a leitura do livro de Lemos e Bairral (2010). O livro explora a geometria dos poliedros estrelados regulares e apresenta diferentes recursos para abordá-los.

A partir da constatação das autoras de que os alunos dessa instituição não conheciam tal teoria e de uma reflexão sobre as práticas propostas no livro, surgiram as seguintes inquietações: como trabalhar a importância e a flexibilidade das definições? Como trabalhar esse conteúdo de forma interessante e divertida?

Como resposta, o presente trabalho consiste em um relato de experiência sobre uma oficina para discutir a precisão que devemos ter com definições e, a partir daí, desenvolver o conceito de poliedros estrelados, sua regularidade e características, utilizando construção com origami. A oficina em questão foi ministrada para 21 alunos de Licenciatura Matemática de uma Universidade Federal, em uma proposta investigativa. Nesse artigo faremos um recorte das atividades desenvolvidas, priorizando a reflexão sobre definições de polígonos e poliedros.

\section{A importância das definições}

Veloso (2006) traz uma discussão interessante sobre a questão histórica das definições no ensino de matemática, em especial da geometria. Durante centenas de anos, toda a geometria foi baseada em Os Elementos de Euclides e, portanto, as definições eram absolutas. Isso era necessário para que os jovens entendessem o caráter hipotético-dedutivo da geometria. Até que alguns matemáticos questionaram essa metodologia. "Dieudoneé chegou a classificar de escroquerie [fraude] o modo de ensinar matemática" (VELOSO, 2006, p. 7).

Ainda sobre definições, Grumbaum (2003) nos ensinou que a palavra poliedro tem significado diferente para pessoas diferentes. É isso que justifica a contagem do número de poliedros regulares ter sido alterada ao longo do tempo. Euclides provou a existência de apenas cinco poliedros regulares. Cauchy, no início do século XIX, provou que eram nove. Por volta de 1920, Petrie e Coxeter encontraram mais três, provando a completude dessa afirmação com doze poliedros regulares. E Grumbaum ainda foi além. Como isso é possível?

As long as different people interpret the concept in different ways there is always the possibility that results true under one interpretation are false with other understandings. As a matter of fact, even slight variations in the definitions of concepts often entail significant changes in results. (GRUNBAUM, 2003, p. 462). 


\section{Trabalhando com as definições}

Para avaliar o conhecimento prévio dos alunos, começamos a oficina com a pergunta que nos serviu de inspiração: existe poliedro regular não convexo?

Alguns alunos opinaram com insegurança, "acho que não". Dúvidas e incertezas eram esperadas. Um aluno respondeu: "a gente sabe que não existe, mas se vocês estão perguntando, é porque deve existir”. E riu. É engraçado perceber a contradição na fala desse aluno. Ele tinha uma resposta para a pergunta, mas achava que não era essa resposta que gostaríamos de ouvir. Outro aluno respondeu com a justificativa esperada, fazendo referência aos Poliedros de Platão como sendo os únicos poliedros regulares, todos convexos. Essa explicação foi apoiada pela maioria dos participantes. Achamos que a definição que estava no inconsciente coletivo é a definição usual do ensino básico, onde lados de polígonos e faces de poliedros não têm interseção, a menos de vértices (polígonos) e vértices e arestas (poliedros). Usando essas definições, os alunos estavam corretos.

Em seguida perguntamos o que é poliedro estrelado. Nenhum dos alunos conhecia ou tinha ouvido falar sobre esses poliedros. Passamos então à revisão de conteúdos, nesta sequência: polígono, polígono convexo, polígono regular, poliedro, poliedro convexo e poliedro regular. Vamos nos ater a esse trabalho, à definição de polígonos e poliedros.

Para trabalhar o conceito de polígono, pedimos aos alunos que escrevessem a sua definição e em seguida reconhecessem, dentre um conjunto de figuras, os polígonos. Depois que os alunos definiram os polígonos em suas fichas, pedimos que lessem em voz alta o que escreveram. Duas respostas podem ser vistas na Figura 1:

Figura 1 - Definição de polígono dada por dois alunos

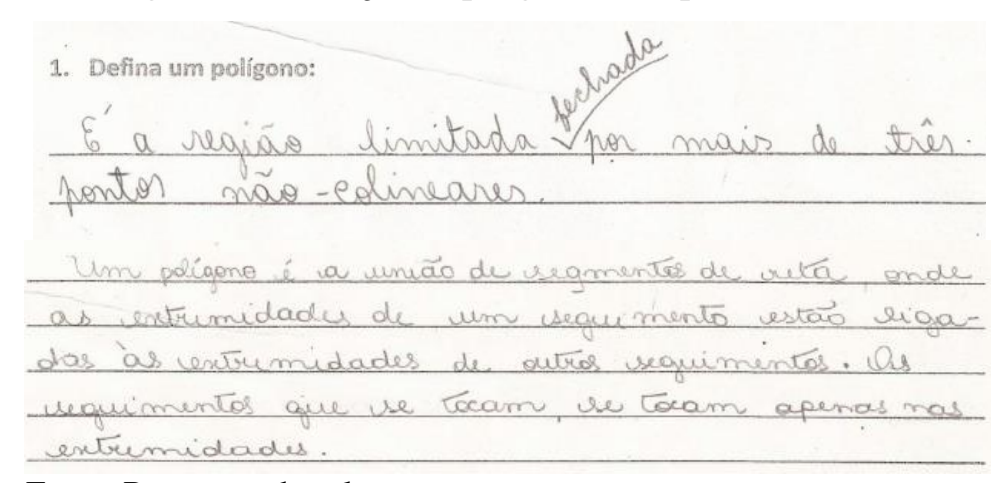

Fonte: Respostas dos alunos.

Essa atividade foi baseada no artigo de Veloso (2006, p.8) que, assim como nós, concluiu: "constatamos que concretamente, mesmo entre os professores de matemática, existem diferentes concepções do que é um polígono". 
As diferentes respostas apresentadas pelos alunos foram cruciais para a discussão sobre flexibilidade com rigorosidade das definições e permitiram desenvolver um clima descontraído onde os alunos se sentissem à vontade para compartilhar opiniões.

Já foram discutidas, em vários artigos, as definições em geometria. A importância de sabermos com quais trabalhamos quando estamos a tratar determinado assunto, as diferentes formas de as abordarmos no ensino, os excessos cometidos quando queremos que os alunos as saibam de forma enciclopédica. (MARQUES, 2008, p.29)

Apesar de enfatizar que o objetivo da atividade não era avaliar se a resposta estava certa ou errada, alguns alunos iniciaram sua fala declarando "não sei se está certo" ou "se estiver errado, corrija". Essas sentenças são reflexos da maneira como se ensina matemática. Nesse sentido, a proposta de uma aula investigativa foi muito importante, embora os alunos não estivessem acostumados a isso.

Depois de longa discussão, chegamos a uma definição comum para polígonos: "um polígono é uma linha poligonal fechada sem auto-interseções, isto é, cada lado tem apenas um ponto comum com o lado anterior e com o seguinte, mas não com os demais" (CARVALHO et al, 2010, p.1).

Para revisar a definição de poliedros, utilizamos outra técnica de investigação, apresentando diferentes sólidos confeccionados em origami por uma das autoras (Figura 2). Os alunos puderam experimentar os objetos para justificar se ele era ou não um poliedro. E ainda, se era ou não convexo e/ou regular. Esta atividade permitiu apresentar o origami modular e explorar algumas possibilidades do origami como recurso para enriquecer a aprendizagem de matemática.

Figura 2 - sólidos confeccionados em origami

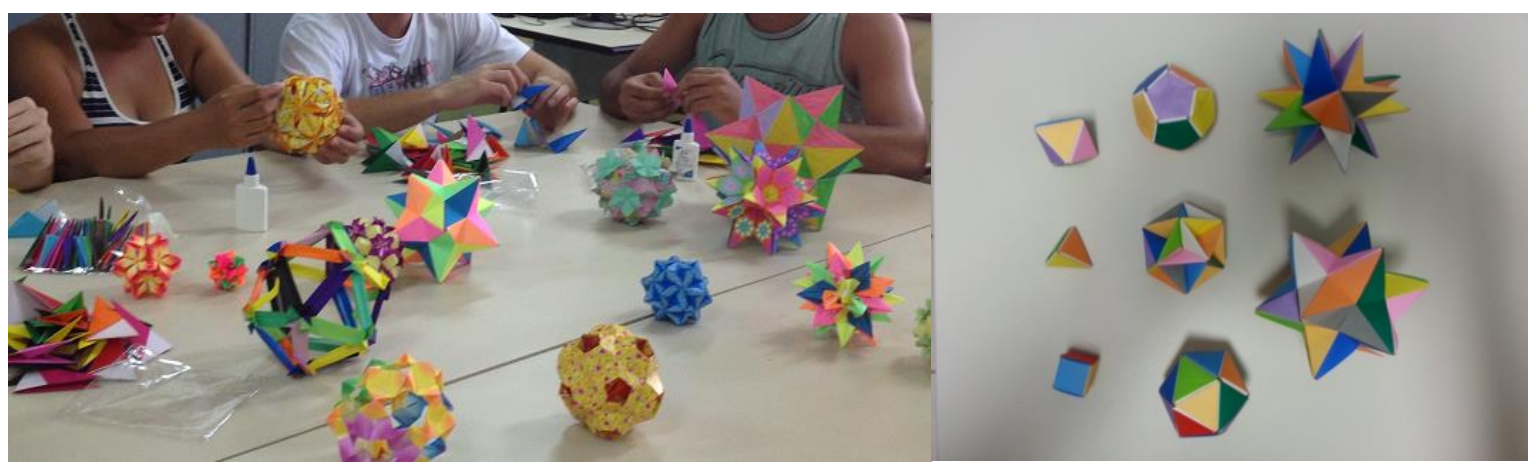

Fonte: Elaborada pelas autoras.

Os origamis chamaram muito a atenção dos alunos por sua beleza estética e diferentes materiais. Os alunos ficaram intrigados com o sólido da Figura 3.

Figura 3 - Sólido não regular feito em origami 


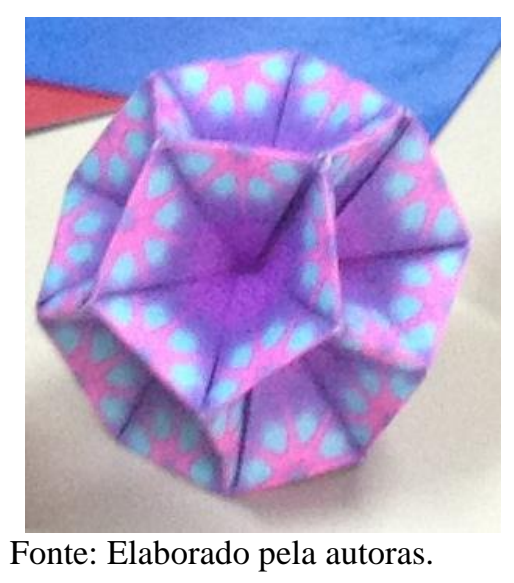

Por sugestão dos alunos, a definição de poliedro foi dada recorrendo à literatura. Foi interessante notar que já aqui os alunos entenderam a importância da definição rigorosa e se utilizaram dela para classificar os sólidos. Podiam ter eles mesmos chegado a essa definição por investigação, mas não seguiram esse caminho. Ainda assim, perceberam que a teoria e a prática estão sempre alinhadas e que uma não sobrevive sem a outra.

Um poliedro pode ser definido como um conjunto finito e conexo de polígonos planos tal que cada lado de cada polígono é comum a outro polígono, e a mais nenhum, de tal forma que os polígonos que estão à volta de um mesmo vértice formam um único circuito. Os polígonos são chamados faces e os seus lados são as arestas do poliedro. (COXETER, 1973, p.4)

Voltando ao sólido da Figura 3, verificamos que ele possui faces com uma aresta que não está conectada a nenhuma outra face (polígono); logo, não se caracteriza como poliedro.

Os alunos concluíram que, assumindo a definição de Coxeter, existem apenas cinco poliedros regulares, todos convexos, os Poliedros de Platão. Essa afirmação foi provada pela primeira vez em Os Elementos de Euclides: "Digo então que exceto as cinco ditas figuras [poliedros de Platão] não será construída outra figura contida por equiláteros e também equiângulos iguais entre si” (EUCLIDES; BICUDO, 2009, p.592).

Em um terceiro momento, aplicamos um questionário com perguntas acerca de polígonos e poliedros estrelados. O questionário previa dois momentos de resposta, antes da apresentação do processo de estrelação, a fim de avaliar o conhecimento a priori dos alunos, e ao final da oficina, a fim de registrar o que foi aprendido. Essa atividade foi baseada no livro de Lemos e Bairral (2010). Ficou claro que nenhum aluno conhecia os termos "estrelado" ou "estrelação" antes da oficina.

A questão que queríamos ressaltar com os alunos é que a definição de polígono e poliedros pode ser flexibilizada, de modo que os polígonos e poliedros estrelados sejam regulares. Basta permitir que lados de um polígono e faces de um poliedro possam se intersectar. Os alunos não imaginavam que isso fosse possível. 
Apresentamos o conceito de estrelação de um polígono prolongando os seus lados e formando novo polígono. Mostramos que nem todo polígono é estrelável (triângulo, quadrado), que alguns apresentam uma falsa estrelação, com a sobreposição de dois polígonos (hexágono) e finalmente mostramos polígonos estrelados, como o pentagrama.

Naturalmente surgiu na turma a dúvida sobre a possibilidade de estrelação dos poliedros. Foi interessante notar a curiosidade dos alunos e a percepção de generalização das definições flexibilizadas assim como ocorria com as definições de Euclides. Utilizamos os sólidos de origami para mostrar alguns poliedros estrelados e trabalhamos o conceito de estrelação de um poliedro, como a interseção do prolongamento de faces. Em seguida, construímos com os alunos o pequeno dodecaedro estrelado, que é a primeira estrelação do dodecaedro, utilizando técnicas de origami modular propostas em Lemos e Bairral (2010). Detalhes desse processo estão descritos em Morosini e Wrobel (2014). Essa atividade foi realizada parcialmente em aula e parcialmente em casa, otimizando o tempo da oficina.

A partir da manipulação do pequeno dodecaedro estrelado foi possível visualizar e contar a quantidade de faces, vértices e arestas que compõem o sólido e verificar que o conceito de regularidade permanecia válido naquele sólido. Os alunos concluíram que existia mais um poliedro regular, além dos de Platão, ou seja, se admitirmos que as faces dos poliedros podem se intersectar sem restrições, existem sólidos regulares não convexos. Essa era a grande descoberta!

Para valorizar essa constatação, apresentamos e analisamos também o grande dodecaedro e o grande dodecaedro estrelado confeccionados em origami. Falamos da existência do grande icosaedro, mostrando-o em figuras. Nesse momento, foi oportuna a apresentação da história e algumas curiosidades sobre os poliedros estrelados regulares, os Poliedros de Kepler-Poinsot.

Ao final da oficina retomamos a pergunta Existe poliedro regular não convexo?, que claramente obteve resposta diferente da apresentada no início. Os alunos foram então apresentados ao Teorema de Existência na forma provada por Cauchy: existem nove poliedros regulares.

\section{Conclusão}

Neste artigo, divulgamos uma experiência investigativa para trabalhar a rigorosidade, mas não unicidade, de definições de polígonos e poliedros. Esse tema é pouco trabalhado nas formações de professores e, consequentemente, quase não se tem notícias dele no ensino básico.

Durante a oficina foi possível aprender que com a flexibilização da definição de poliedro, existem nove poliedros regulares, os cinco poliedros de Platão (convexos) e os quatro poliedros de Kepler-Poinsot (não convexos). Esse teorema foi provado por Cauchy no século XIX. 
Quando perguntado aos alunos se eles seriam capazes de imaginar sólidos como aqueles, a resposta negativa foi unânime. Estamos condicionados a recorrer a imagens cotidianas e o origami proporcionou extrapolar essa linearidade de pensamento.

As atividades aqui apresentadas não trazem uma receita pronta, mas uma ideia que pode ser construída em vários cenários, agregando novas informações e investigações. Perceber a surpresa, o encantamento, o engajamento e a motivação dos alunos sugere que estamos no caminho certo para formar professores críticos e preocupados com aquilo que vão ensinar e com a maneira como vão ensinar.

\section{Referências}

CARVAlHO, P. C. et al.. A matemática no ensino médio. Vol 2. Rio de Janeiro, SBM, 2010. (Coleção Professor de Matemática)

COXETER, H. S. M. Regular Polytopes. London: Ed. Dover, 1973.

EUCLIDES; BICUDO, I. (Trad.). Os Elementos de Euclides. São Paulo: Fundação Editora da UNESP, 2009.

GRÜNBAUM, B. Are Your Polyhedra The Same as My Polyhedra? Em Aronov, B.; Basu, S.; Pach, J.;Sharir, M. (editores). Discrete and Computational Geometry: The Goodman-Pollack Festschrift. Springer-Verlag, p. 461-588, 2003.

LEMOS, W. G.; BAIRRAL, M. A. Poliedros Estrelados no currículo do ensino médio. Rio de Janeiro: Edur, 2010.

MARQUES, P. M. Poliedros Regulares. Educação e Matemática, Lisboa, 2008. http://www.apm.pt/files/_29-32_hq_482c13d3653bf.pdf . Acesso em 01 de out de 2012.

MOROSINI, T. H. N. ; WROBEL, J. S. Poliedros Estrelados e Origami: uma experiência na formação de professores. Revista Eletrônica Sala de Aula em Foco, v. 3, n.1 p. 31-41, 2014. VELOSO, E.. Sobre as definições (I). Educação e Matemática. APM. p. 7-9. nov-dez 2006. http://www.apm.pt/files/_19-22_lq_46b1efa84e3d8.pdf. Acesso em 01 de out de 2012.

Submetido em dezembro de 2014

Aprovado em maio de 2015 